\title{
First records of Buchonomyia thienemanni Fittkau (Diptera: Chironomidae) from the Czech Republic
}

\author{
Patrick Ashe ${ }^{1}$, Joel Moubayed-Breil ${ }^{2}$ and Daniel Vondrák ${ }^{3}$ \\ 133 Shelton Drive, Terenure, Dublin 12, Ireland.E-mail: patrick.ashe@upcmail.ie \\ ${ }^{2}$ Marine \& Freshwater Biology, 10 rue des Fenouils, 34070 Montpellier, France. \\ E-mail:jm.aquabiol@neuf.fr \\ ${ }^{3}$ Institute for Environmental Studies, Faculty of Science, Charles University in Prague, Benátská street 2, \\ 12843 Prague 2, Czech Republic.E-mail: daniel.vondrak@natur.cuni.cz
}

Buchonomyia thienemanni Fittkau is recorded from the Czech Republic for the first time based on specimens collected in samples from a section of the River Dyje in Podyjí National Park (Moravia) and the Litavka River in the Brdy Mountains (Bohemia).

Buchonomyia thienemanni Fittkau was originally described based on a single adult male collected at an altitude of 800 metres in the Wasserkuppe spring region of the River Fulda in Germany (Fittkau 1955). After a long gap of twenty one years a second record was published when adults were found by the River Flesk, S. W. Ireland (Murray 1976). The pupal stage was subsequently discovered and described based on associated material from the River Flesk (Murray and Ashe 1981). The adult stage is more elusive and most records of the species in the western Palaearctic (Europe, North Africa, Iran) are based on the pupal exuviae which can be easily collected in drift samples or in foam samples from rivers.

While attending the $19^{\text {th }}$ International Symposium on Chironomidae in České Budějovice (17-22 August 2014) the authors took part in the two day post conference tour. On the $21^{\text {st }}$ August the tour group walked for a few hours through a section of the Podyjí National Park. The group eventually reached an elevated area with an excellent view overlooking the Šbes Vineyard and a section of the River Dyje with extensive surrounding natural woodland (Fig. 1). This woodland covers the nearby mountains and extends down to both banks of the river. When the first two authors (PA and JM-B) saw this section of pristine river we immediately suspected that $B$. thienemanni could be found there. We followed the track down from the vineyard to the River Dyje and crossed over the footbridge. On the south bank of the river, about 50 metres below the footbridge, it was possible to directly access the river where the bank was lower. There was quite a lot of river foam which had accumulated behind obstacles such as emergent rocks and tree branches. River foam, forming over several hours or days, may accumulate large numbers of chironomids (pupal exuviae, pharates and drowned adults) and is easily collected with a drift net. One large foam sample was collected just beside the river bank and a more extensive sample was collected further out in shallow water (Fig. 2) which included foam and material washed from aquatic plants and stones. When the large foam sample was being processed for preservation pupal exuviae of $B$. thienemanni were observed and later examination revealed the presence of a total of 98 pupal exuviae and one drowned adult male. The use of drift or hand nets, to collect foam samples, to determine whether or not $B$. thienemanni occurred in the River Dyje proved immediately successful.

Ten days later, on the 31 August 2014, the junior author (DV) was collecting samples from the Litavka

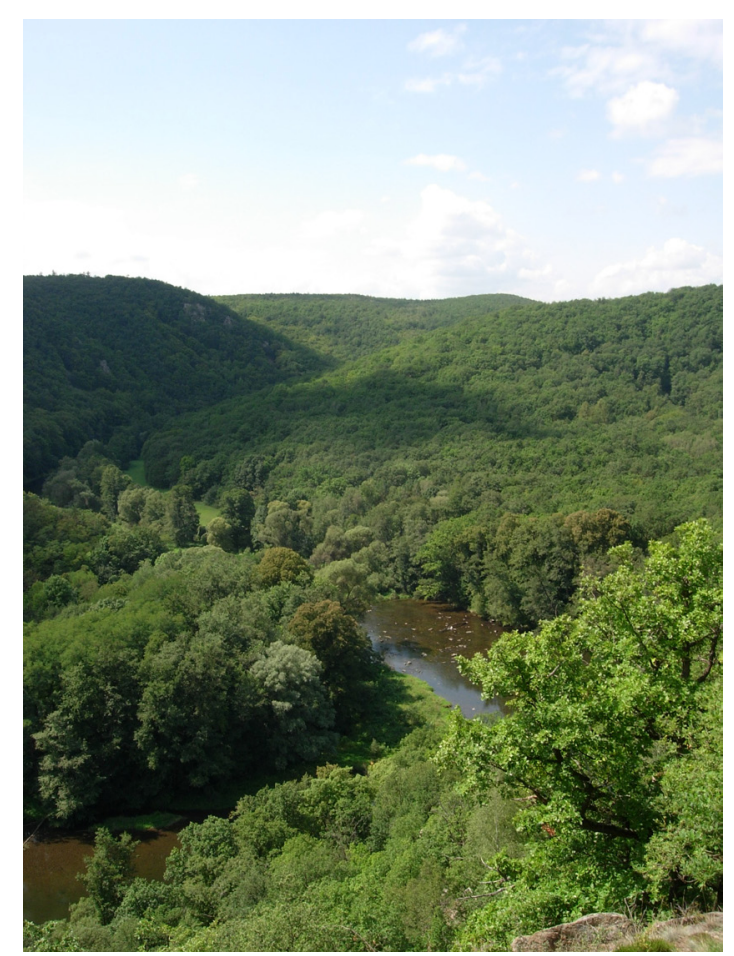

Figure 1. Elevated area overlooking a section of the River Dyje (upstream of sampling site) with extensive natural woodland. Photo: P. Ashe. 


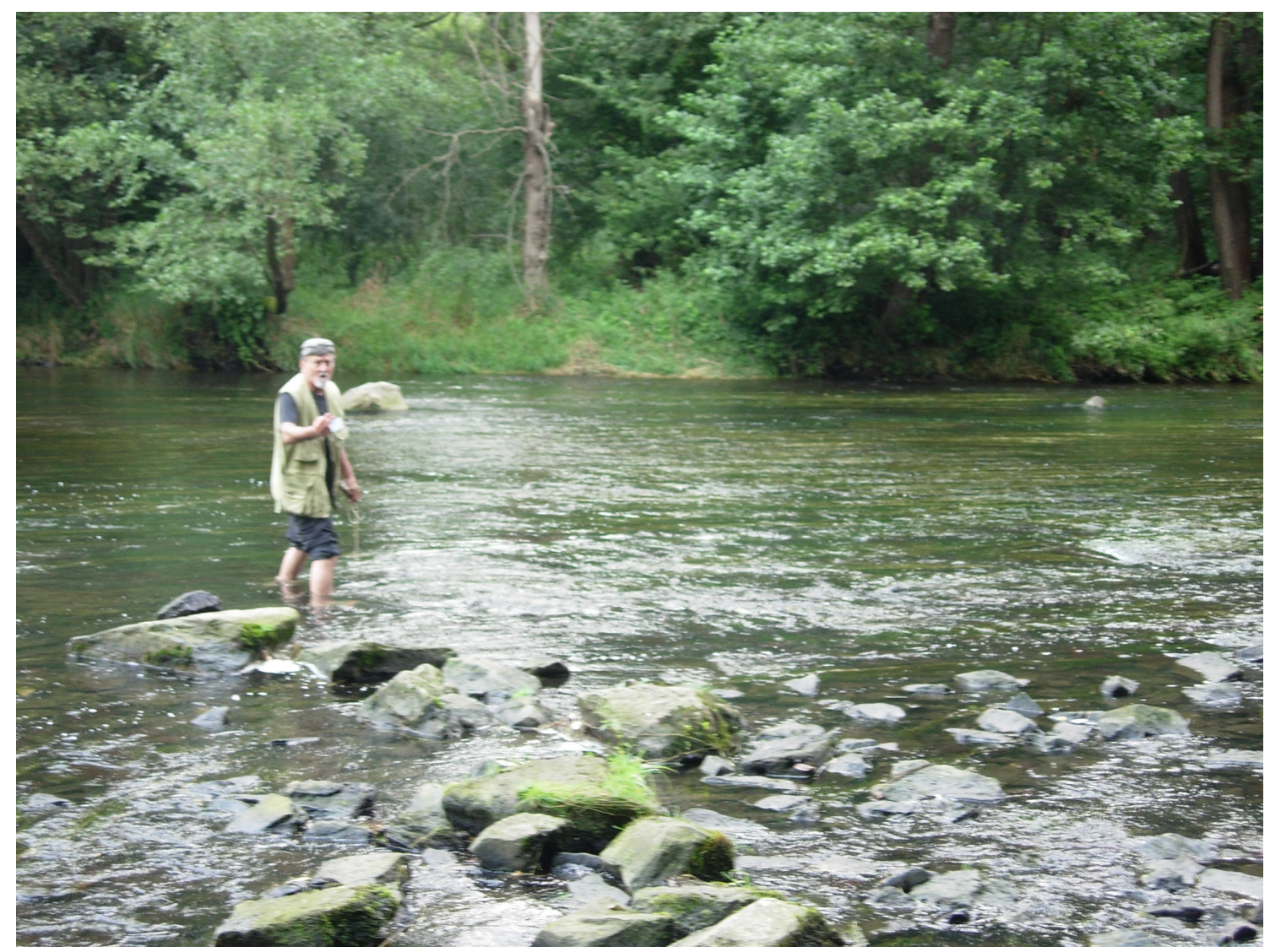

Figure 2. Joel Moubayed-Breil sampling, where B. thienemanni was found, in the River Dyje about 50 metres downstream of the footbridge. Photo: P. Ashe.

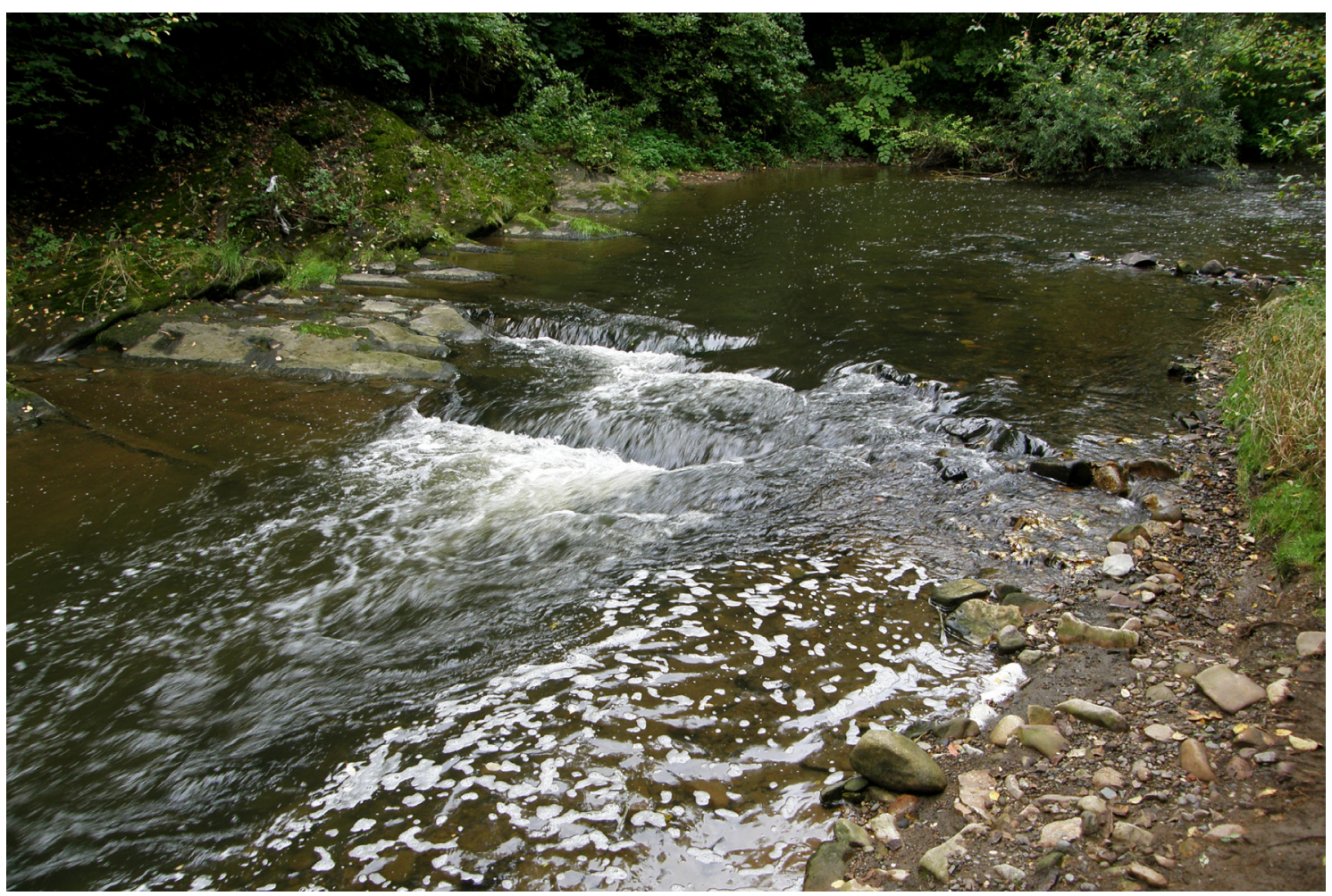

Figure 3. Litavka River, B. thienemanni sampling site. Photo: D. Vondrák. 
River (Figs 3, 4) which rises in the Brdy Mountains in Bohemia. A hand net was used and pupal exuviae of $B$. thienemanni were found but they were not as numerous in the Litavka sample as those from the River Dyje samples.

Full details of the records are as follows:

CZECH REPUBLIC: Moravia: 21 August 2014, circa $1.2 \mathrm{~km}$ north of Hnanice, 50 metres downstream of footbridge, south bank of the River Dyje, Podyjí National Park, $48^{\circ} 48^{\prime} 39^{\prime}$ N, $15^{\circ} 58^{\prime} 40^{\prime}$ E, leg. P. Ashe \& J. Moubayed-Breil; Bohemia: $31 \mathrm{Au}-$ gust 2014, circa $0.5 \mathrm{~km}$ south of road bridge in Lochovice at $314 \mathrm{~m}$ a.s.1., next to the soccer field, west bank of the Litavka River, Brdy Mountains, $49^{\circ} 50^{\prime}$ 54” N, $13^{\circ} 58$ ' 53” E, leg. D. Vondrák.

Voucher specimens from the River Dyje have been deposited in the collections of the Zoologische Staatssammlung, Munich, Germany (3 pupal exuviae and 1 drowned adult male) and the National Museum of Ireland, Dublin, Ireland (4 pupal exuviae)- the remaining material from this site is in the respective collections of the two senior authors (PA and JM-B). Material of $B$. thienemanni from the Litavka River, collected by the junior author (DV), is in the collec-

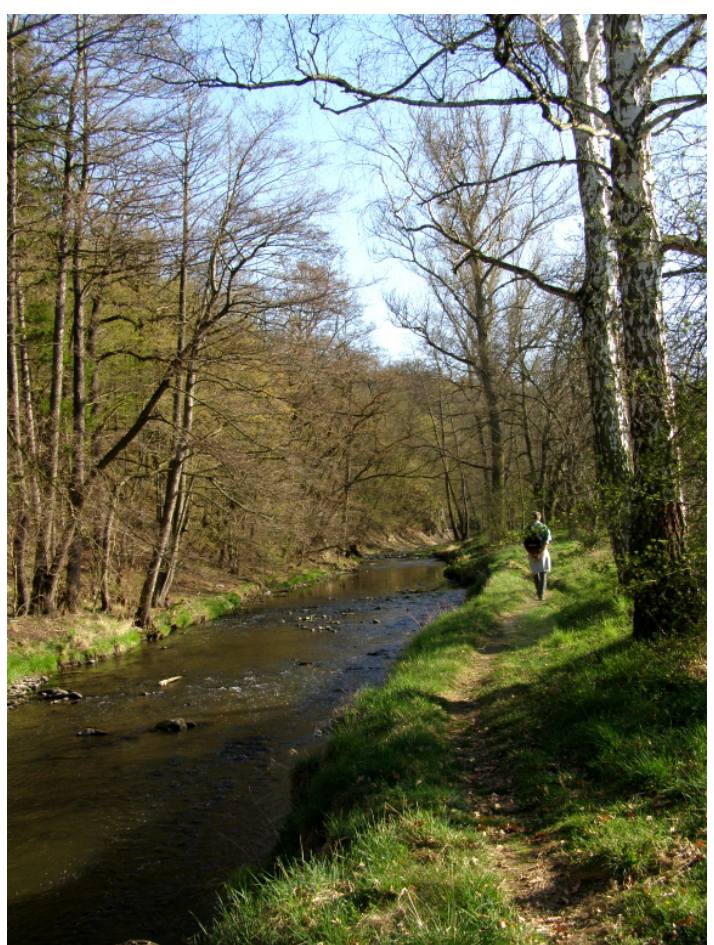

Figure 4. Litavka River, 250 metres upstream of sampling site. Photo: D. Vondrák. tion of the second author (JM-B).

The locality data detailed above from the River Dyje in Moravia and the Litavka River in Bohemia are the first records of $B$. thienemanni from the Czech Republic.

\section{Acknowledgements}

We are grateful to the organizing committee of the Chironomid Symposium in České Budějovice who arranged the post-conference tour which included the River Dyje in Podyjí National Park.

\section{References}

Fittkau, E. J. 1955. Buchonomyia thienemanni n. gen. n. sp. Chironomidenstudien IV (Diptera: Chironomidae). - Beiträge zur Entomologie 5: 403-414.

Murray, D. A. 1976. Buchonomyia thienemanni Fittkau (Diptera, Chironomidae), a rare and unusual species recorded from Killarney, Ireland. - Entomologist's Gazette 27: 179-180.

Murray, D. A. and Ashe, P. 1981. A description of the pupa of Buchonomyia thienemanni Fittkau, with notes on its ecology and on the phylogenetic position of the subfamily Buchonomyiinae (Diptera, Chironomidae). - Spixiana 4: 55-68. 